\title{
A low luminosity state in the massive X-ray binary SAX J0635+0533
}

\author{
Sandro Mereghetti ${ }^{1}$, Nicola La Palombara ${ }^{1}$ \\ INAF, Istituto di Astrofisica Spaziale e Fisica Cosmica Milano, via E. Bassini 15, I-20133 Milano, Italy \\ Received: February 25, 2009 / Accepted: June 2, 2009

\begin{abstract}
The X-ray pulsar SAX J0635+0533 was repeatedly observed with the XMM-Newton satellite in 2003-2004. The precise localization provided by these observations confirms the association of SAX J0635+0533 with a Be star. The source was found, for the first time, in a low intensity state, a factor $\sim 30$ lower than that seen in all previous observations. The spectrum, well fitted by an absorbed power law with photon index $\sim 1.7$ and $\mathrm{N}_{H}=1.2 \times 10^{22} \mathrm{~cm}^{-2}$, was compatible with that of the high state. The low flux did not allow the detection of the pulsations at $33.8 \mathrm{~ms}$ seen BeppoSAX and RXTE data. In view of the small luminosity observed in 2003-2004, we reconsider the peculiarities of this source in both the accretion and rotation powered scenarios.
\end{abstract}

Key words. stars: individual: SAX J0635+0533 - X-rays: binaries

\section{Introduction}

The X-ray source SAX J0635+0533 was discovered with BeppoSAX in October 1997 (Kaaret et al. 1999) during a search for counterparts of unidentified gamma-ray sources (Thompson et al. 1995). Its $2-10 \mathrm{keV}$ flux of $1.2 \times 10^{-11}$ erg $\mathrm{cm}^{-2} \mathrm{~s}^{-1}$, hard power law spectrum (photon index $\sim 1.5$ ) extending to $40 \mathrm{keV}$, and positional coincidence with a $\mathrm{V}=$ 12.8 star of Be spectral type, immediately suggested to classify SAX J0635+0533 as an accreting high mass X-ray binary. The subsequent discovery of X-ray pulsations at $33.8 \mathrm{~ms}$ (Cusumano et al. 2000) in the BeppoSAX data, makes this object quite peculiar and raises some problems for the accretion scenario. In fact, if the X-ray emission is powered by accretion on the neutron star surface, in order to avoid the propeller effect (see, e.g., Campana et al. (1995)), the magnetic field in SAX J0635+0533 must be about three orders of magnitude smaller than expected in a typical high mass X-ray binary.

The pulse frequencies measured with RXTE in 1999, about two years after the source discovery, indicated an orbital modulation with a period of about 11 days and set a lower limit on the long term spin-down $\dot{P}>3.8 \times 10^{-13} \mathrm{~s} \mathrm{~s}^{-1}$ Kaaret et al. 2000). Such a high $\dot{P}$ in a rapidly spinning neutron star implies a large rotational energy loss, $\dot{E}_{\text {rot }}=10^{45} 4 \pi^{2} \dot{P} / P^{3}=4 \times 10^{38}$ erg $\mathrm{s}^{-1}$, capable to power the observed X-ray luminosity without the need of invoking mass accretion. In this interpretation, SAX J0635+0533 would resemble other binary systems composed of a fast pulsar orbiting a massive star, such as PSR B1259-63 (Johnston et al. 1992), in which the X-ray emission is thought to originate in the shock between the pulsar's rel-

Send offprint requests to: S. Mereghetti, sandro@iasf-milano.inaf.it ativistic wind and that of the companion star. The failure to detect radio pulsations from SAX J0635+0533 (Nicastro et al. 2000), does not rule out this scenario, since beaming and/or absorption effects might render the radio emission unobservable.

Here we report the results of XMM-Newton observations, carried out in 2003-2004, during which SAX J0635+0533 was detected at a very low flux level, the smallest ever seen from this source.

\section{Observations and data analysis}

SAX J0635+0533 was observed by XMM-Newton with ten different pointings between 2003 September $11^{\text {th }}$ and 2004 April $14^{\text {th }}$. The three EPIC focal plane cameras (Turner et al. 2001; Strüder et al.2001) were active during these pointings. The two MOS cameras were operated in the standard Full Frame mode (time resolution $2.6 \mathrm{~s}$ ), in order to cover the whole $30^{\prime}$ field-ofview. The $p n$ camera was operated in Timing mode, providing a time resolution of $30 \mu \mathrm{s}$, but without imaging information. For each observation and focal plane camera the thin filter was used.

For each pointing we retrieved from the XMM-Newton archive the pps files produced by pipeline processing system, which is operated by the Survey Science Center. Since only Timing mode data were obtained with the $p n$ camera, we concentrated on the MOS data to study the source properties. Due to the faintness of SAX J0635+0533 during these observations, it was not possible to see the $33.8 \mathrm{~ms}$ pulsations in the $p n$ camera data. For each observation, we looked for possible periods of high instrumental background, caused by flares of soft protons with energies less than a few hundred $\mathrm{keV}$. To this aim, we 
selected only single and double events (PATTERN $\leq 4)$ with energies greater than $10 \mathrm{keV}$ and recorded in the peripheral CCDs. Then, we set a count-rate threshold for good time intervals (GTI) at $0.5 \mathrm{cts} \mathrm{s}^{-1}$. By selecting only events within GTIs we finally obtained a "clean" event list for each MOS data set. The dates and effective exposure times (after soft-proton rejection) of the observations are listed in Table 1

After merging the event lists of the two MOS cameras, we accumulated an image of the field-of-view comprising all the observations. This clearly showed a source, with a count rate of $(4.3 \pm 0.3) \times 10^{-3}$ counts s ${ }^{-1}$ in each camera, at the coordinates R.A. $=06^{h} 35^{m} 18.3^{s}$, Dec. $=+05^{\circ} 33^{\prime} 06.3^{\prime \prime}(\mathrm{J} 2000)$. This position was obtained after the astrometric correction of the X-ray coordinates of the detected sources, based on the positions of five optical counterparts found in the Guide Star Catalogue (Lasker et al. 2008). The final uncertainty on the source position is $1^{\prime \prime}$ at $90 \%$ c.l. (including statistical and systematic errors). The XMM-Newton localization confirms the association with the star proposed by Kaaret et al. (1999) on the basis of an estimated $4 \%$ probability of finding by chance a Be star in the $30^{\prime \prime}$ radius error circle determined with BeppoSAX (see Fig. 11).

We extracted the source spectra by selecting events in a circular region with a small radius $\left(10^{\prime \prime}\right)$ in order to minimize the background contribution. The background spectra for the two $M O S$ cameras were accumulated from large circular areas with no sources and radii of $100^{\prime \prime}$. We generated ad hoc redistribution matrices and ancillary files using the SAS tasks rmfgen and arfgen, respectively. In order to ensure the applicability of the $\chi^{2}$ statistics, all spectra were rebinned with a minimum of 30 counts per bin, and fitted in the energy range 0.3$10 \mathrm{keV}$ using XSPEC 11.3.2. We checked that separate fits of the two spectra gave consistent results, therefore we analysed them simultaneously, in order to increase the count statistics, imposing common spectral parameters for the two spectra and introducing a free relative normalization factor between them, to account for possible differences in the instrument crosscalibration. We found a 1.02 cross-normalization factor between the MOS2 and the MOS1 camera.

A good fit was obtained with an absorbed powerlaw (Fig[2), yielding a hydrogen column density $N_{\mathrm{H}}=$ $\left(1.17_{-0.35}^{+0.49}\right) \times 10^{22} \mathrm{~cm}^{-2}$ and a photon index $\Gamma=1.74_{-0.31}^{+0.43}$. The absorbed flux in the energy range $0.2-12 \mathrm{keV}$ is $f_{\mathrm{X}} \sim$ $1.45 \times 10^{-13} \mathrm{erg} \mathrm{cm}^{-2} \mathrm{~s}^{-1}$, while the corresponding unabsorbed flux is $\sim 2.55 \times 10^{-13} \mathrm{erg} \mathrm{cm}^{-2} \mathrm{~s}^{-1}$. Acceptable fits were also obtained with a thermal bremsstrahlung $(k T=9.2$ $\mathrm{keV}$ ) and with a blackbody ( $k T \sim 1 \mathrm{keV})$.

To study the source variability, we analyzed separately the ten data-sets. For each of them we performed a detailed source detection in five energy bands $(0.2-0.5,0.5-1,1-2,2-$ 4.5 and $4.5-12 \mathrm{keV}$ ), applying the same procedure and parameters used by the XMM-Newton SSC to produce the XMMNewton serendipitous source catalogue (Watson et al. 2009). The source detection was performed simultaneously on both $M O S$ data sets and using the corresponding exposure maps which account for spatial quantum efficiency variations, mirror vignetting, and effective field of view. The threshold value for the detection likelihood was set to 5 for the likemin parameter

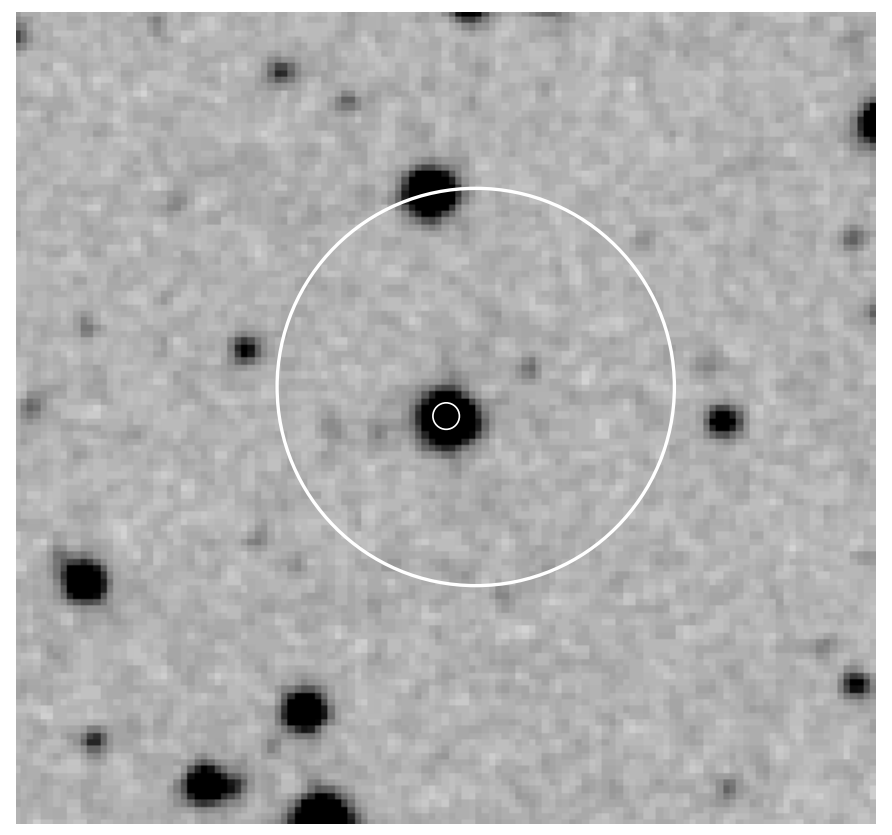

Fig. 1. Optical image of a $2^{\prime} \times 2^{\prime}$ field around the position of SAX J0635+0533 from the Digitized Sky Survey (B filter). The large circle is the BeppoSAX error region with a radius of $30^{\prime \prime}$ (Cusumano et al. 2000). The small circle ( $2^{\prime \prime}$ radius) is the XMM-Newton position. North is to the top, East to the left.

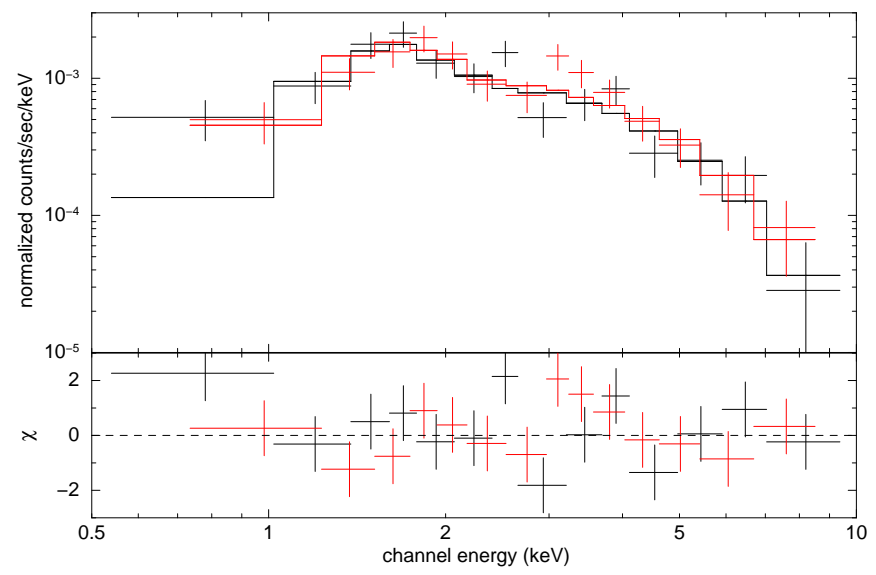

Fig. 2. Top panel: average spectrum of SAX J0635+0533 with the best-fit power-law model. The spectra of the MOS1 and MOS2 cameras are shown in black and red, respectively. Bottom panel: data-model residuals, in units of $\sigma$.

of the $S A S$ task eboxdetect and to 6 for the mlmin parameter of the $S A S$ tasks emldetect and esensmap. We emphasize that these values imply a rather loose constraint on the source significance in order to be detected; they allow to detect even very weak sources, with a low (i.e. $\simeq 3$ ) signal to noise ratio. In this way we aim to check if SAX J0635+0533 is even only marginally detected in any observation. On the other hand, in our detection procedure we used 'ad hoc' energy conversion factors $(E C F)$ to convert the measured count rates of the detected sources (both in each of the five energy bands and in the total $0.2-12 \mathrm{keV}$ band) into the corresponding energy flux. 
They were derived based on the best-fit power-law model of the source average spectra.

Based on this analysis, we found that SAX J0635+0533 was detected only in six observations, as reported in Table 1 The upper limits on the count rate (at the confidence level corresponding to a detection likelihood $L=6$ ) are obtained from the sensitivity maps at the source position.

The long term light curve of SAX J0635+0533 is plotted in Fig. 3. In September-October 2003 the source flux varied by at least a factor 10. Although the observations are not continuous, they suggest an outburst lasting about three weeks, with a rise time of only a few days to a maximum flux of $\sim 5 \times 10^{-13}$ erg $\mathrm{cm}^{-2} \mathrm{~s}^{-1}$, followed by a similarly rapid decay. A similar flux level was observed again six months later. Comparing the hardness ratios measured in the different observations, we found some evidence for a slight spectral hardening correlated with the source intensity (Table 1, Fig. 4).

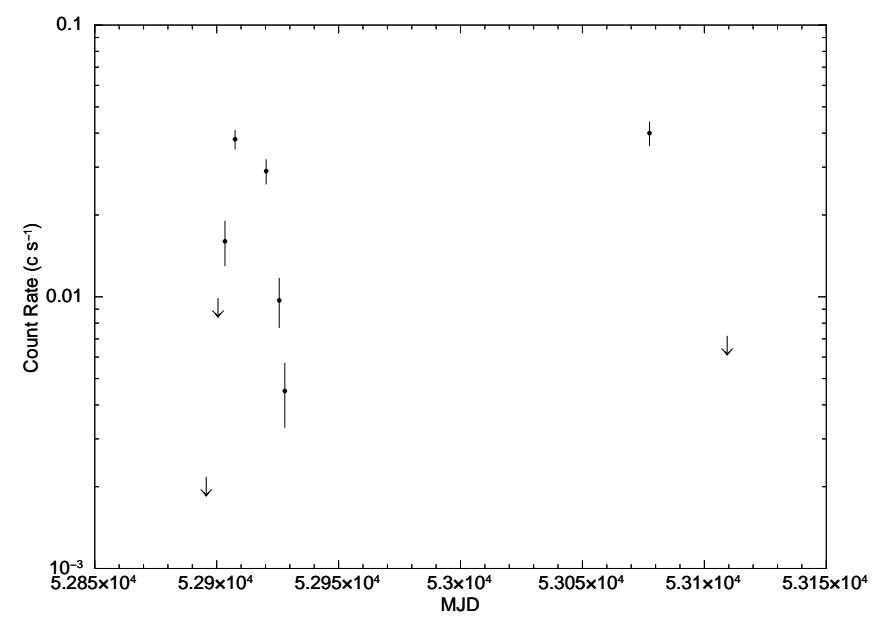

Fig. 3. Light curve of SAX J0635+0533. The count rates refer to the $0.2-12 \mathrm{keV}$ and to the sum of $2 \mathrm{MOS}$. The data of the first two observations have been merged. The upper limits (obtained with a threshold in detection likelihood $L=6$ ) correspond to a $\sim 3 \sigma$ confidence level.

\section{Discussion}

The maximum flux we observed for SAX J0635+0533, 4.2 $\times 10^{-13} \mathrm{erg} \mathrm{cm}^{-2} \mathrm{~s}^{-1}$ in the $2-10 \mathrm{keV}$ range, is a factor $>$ 30 smaller than that measured at the time of the BeppoSAX discovery in $1997\left(1.2 \times 10^{-11} \mathrm{erg} \mathrm{cm}^{-2} \mathrm{~s}^{-1}\right.$, Kaaret et al. (1999)). To our knowledge, this is the lowest flux ever reported for SAX J0635+0533. The upper limits of the XMM-Newton pointings of September 2003 imply an even smaller flux.

The distance of SAX J0635+0533 is not well constrained. The range 2.5-5 kpc was estimated by Kaaret et al. (1999) from the properties of the proposed optical counterpart. We also note that a distance much in excess $5 \mathrm{kpc}$ is unlikely, considering the location of SAX J0635+0533 in the Galactic anti-center direction. In the following discussion, where we consider the two alternative possibilities for the origin of the observed X-ray

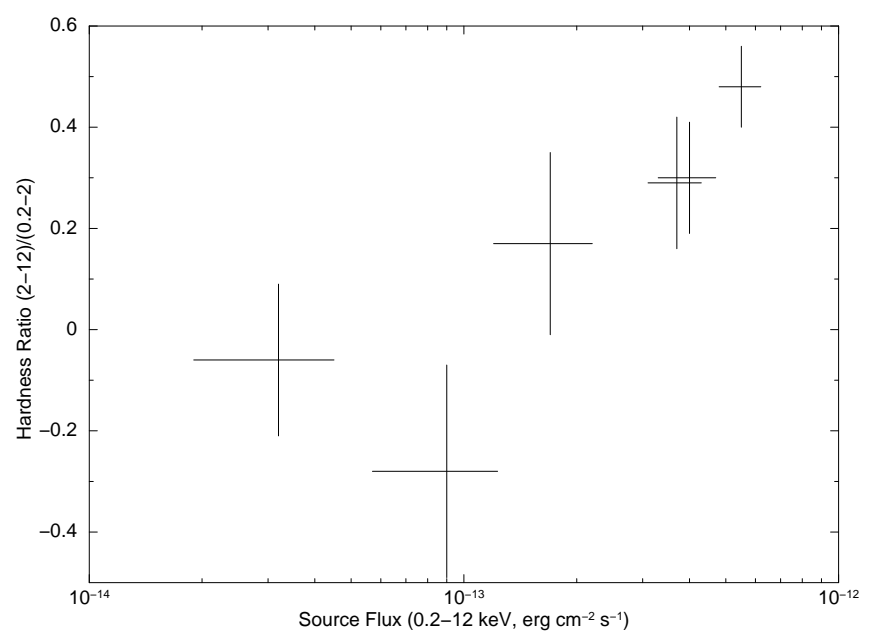

Fig. 4. Hardness ratio versus source flux. The hardness ratio is defined as $(\mathrm{H}-\mathrm{S}) /(\mathrm{H}+\mathrm{S})$, where $\mathrm{H}$ and $\mathrm{S}$ are the source count rates in the hard $(\mathrm{H}=2-12 \mathrm{keV})$ and soft $(\mathrm{S}=0.2-2 \mathrm{keV})$ energy ranges.

emission, we conservatively normalize the relevant quantities to a distance $\mathrm{d}_{5}$ of $5 \mathrm{kpc}$.

\subsection{Accretion powered $X$-ray emission}

Neutron stars accreting from Be star companions constitute the large majority of the high mass X-ray binary systems present in the Galaxy. Also in view of our improved localization of SAX $\mathrm{J} 0635+0533$, it is natural to first discuss this possibility.

Already at the time of its discovery, it was noticed that the luminosity of SAX J0635+0533 was relatively small compared to classical Be/neutron stars systems. The persistent sources of this class have $\mathrm{X}$-ray luminosity of $10^{36}-10^{37} \mathrm{erg} \mathrm{s}^{-1}$. This is also the luminosity typically reached during the outbursts of transient Be/neutron stars systems, which comprise the majority of this population. With the advent of more sensitive observations a number of persistent Be binaries with lower luminosity, $\sim 10^{34} \mathrm{erg} \mathrm{s}^{-1}$, have also been discovered (see, e.g. Reig \& Roche (1999); La Palombara \& Mereghetti (2006, 2007)). Our observations indicate for SAX J0635+0533 an average luminosity, a few $10^{33} \mathrm{~d}_{5}^{2} \mathrm{erg} \mathrm{s}^{-1}$, much smaller than these values, and provide an upper limit as low as $3 \times 10^{32} \mathrm{~d}_{5}^{2}$ $\mathrm{erg} \mathrm{s}^{-1}$ in mid September 2003. If we further consider that the source could well be closer than $5 \mathrm{kpc}$, we are faced with an even smaller luminosity.

The new data clearly indicate that SAX J0635+0533 is a transient source, but it differs from the other Be systems for its low luminosity both during the "high state" and in "quiescence". The non detection in September 2003 allows us to set an upper limit on the mass accretion rate of $1.5 \times 10^{12} \mathrm{~g} \mathrm{~s}^{-1}$. This limit applies assuming that the accretion flow proceeds down to the neutron star surface, which is very unlikely if indeed the neutron star is rotating at $33.8 \mathrm{~ms}$. In the presence of the neutron star magnetic field, different scenarios preventing, or reducing, the accretion rate onto the neutron star surface can occur (see, for example, Campana et al. (1998)). For 
Table 1. Flux and luminosity values of SAX J0635+0533 in the individual observations.

\begin{tabular}{crrrr}
\hline $\begin{array}{c}\text { Start Observation } \\
\text { date - UT }\end{array}$ & $\begin{array}{r}\text { Net exposure } \\
(\mathrm{ks})\end{array}$ & $\begin{array}{r}\text { Absorbed flux }(0.2-12 \mathrm{keV}) \\
\left(\mathrm{erg} \mathrm{cm}^{-2} s^{-1}\right)\end{array}$ & $\begin{array}{r}\text { Luminosity } \\
\left(\mathrm{erg} \mathrm{s}^{-1}\right)\end{array}$ & $\begin{array}{r}\text { Hardness } \\
\text { Ratio }^{(b)}\end{array}$ \\
\hline 2003-09-11 13:22:41 & 8.1 & $<6.1 \times 10^{-14}$ & $<3.03 \times 10^{32}$ & - \\
2003-09-15 18:47:20 & 5.1 & $<7.8 \times 10^{-14}$ & $<3.88 \times 10^{32}$ & - \\
2003-09-18 13:17:46 & 6.8 & $<2.2 \times 10^{-13}$ & $<11.1 \times 10^{32}$ & - \\
2003-09-21 09:53:21 & 3.7 & $(1.7 \pm 0.5) \times 10^{-13}$ & $(8.5 \pm 0.5) \times 10^{32}$ & $0.17 \pm 0.18$ \\
2003-09-25 12:45:02 & 5.0 & $(5.5 \pm 0.7) \times 10^{-13}$ & $(27.6 \pm 3.4) \times 10^{32}$ & $0.48 \pm 0.08$ \\
2003-10-08 07:29:55 & 2.8 & $(3.7 \pm 0.6) \times 10^{-13}$ & $(18.6 \pm 2.4) \times 10^{32}$ & $0.29 \pm 0.13$ \\
2003-10-13 15:30:19 & 4.6 & $(9.0 \pm 3.3) \times 10^{-14}$ & $(4.5 \pm 1.6) \times 10^{32}$ & $-0.28 \pm 0.21$ \\
2003-10-15 22:34:19 & 5.9 & $(3.2 \pm 1.3) \times 10^{-14}$ & $(1.6 \pm 0.7) \times 10^{32}$ & $-0.06 \pm 0.15$ \\
2004-03-13 11:44:58 & 2.7 & $(4.0 \pm 0.7) \times 10^{-13}$ & $(20.7 \pm 3.3) \times 10^{32}$ & $0.30 \pm 0.11$ \\
2004-04-14 08:04:48 & 4.5 & $<1.6 \times 10^{-13}$ & $<8.0 \times 10^{32}$ & - \\
\hline
\end{tabular}

${ }^{(a)}$ In the energy range $0.2-12 \mathrm{keV}$, corrected for the absorption, and assuming a distance of $5 \mathrm{kpc}$.

(b) Ratio between the source count rates in the hard $(\mathrm{H}=2-12 \mathrm{keV})$ and soft $(\mathrm{S}=0.2-2 \mathrm{keV})$ energy ranges, defined as $(\mathrm{H}-\mathrm{S}) /(\mathrm{H}+\mathrm{S})$.

such a short spin period and low luminosity, the direct accretion regime, in which the magnetospheric radius is smaller than the corotation radius and hence the magnetic centrifugal barrier is open, requires a magnetic field smaller than $\sim 10^{8} \mathrm{G}$. This field is at least three orders of magnitude lower than that expected in a young neutron star with a Be companion. In fact all other accreting pulsars of this class have much longer spin periods. The only exception is the recurrent transient A 0538-66 which rotates at $69 \mathrm{~ms}$ (Skinner et al. 1982). Note that the pulsations in this systems were only detected during a bright outburst reaching a luminosity of $\sim 10^{39} \mathrm{erg} \mathrm{s}^{-1}$ (Skinner et al. 1982), implying a magnetic field of $\sim 10^{11} \mathrm{G}$. Although smaller than the average, such a field is not implausible. Furthermore it is consistent with the interpretation of the unpulsed quiescent luminosity of A $0538-66$ (several $10^{33} \mathrm{erg} \mathrm{s}^{-1}$ ) as accretion halted at the centrifugal barrier (Campana et al. 1995).

If SAX J0635+0533 has a typical magnetic field, the low luminosity observed with XMM-Newton could be due to mass accretion stopped at the magnetospheric radius. Assuming for simplicity spherically symmetric accretion, and a neutron star with mass $1.4 M_{\odot}$ and radius $10^{6} \mathrm{~cm}$, gives in this case an X-ray luminosity of $\sim 2 \times 10^{32} B_{12}^{-4 / 7} \dot{M}_{15}^{9 / 7} \mathrm{ergs} \mathrm{s}^{-1}\left(\mathrm{~B}_{12}\right.$ is the magnetic field in units of $10^{12} \mathrm{G}$ and $M_{15}$ the accretion rate in units of $10^{15} \mathrm{~g} \mathrm{~s}^{-1}$; see, e.g., Campana et al. (1998)). However, the higher luminosity state observed in the past with BeppoSAX and RossiXTE cannot be explained in the same way due to the presence of pulsations with a relatively high pulsed fraction, which are not expected when the magnetic centrifugal barrier operates.

In conclusion, the difficulties already pointed out in interpreting SAX J0635+0533 as a typical accretion powered Be binary (Kaaret et al. 2000; Nicastro et al. 2000) are reinforced by the low luminosity reported here. Of course, the properties of this system would fit better in this scenario if the fast periodicity were disproved by further observations.

\subsection{Rotation powered $X$-ray emission}

The alternative interpretation of SAX J0635+0533 is that of a rotation powered neutron star, whose X-ray emission derives from the shock between the relativistic pulsar wind and the companion's wind. The large luminosity difference between our data and the previous observations could be due to varying shock conditions in a very eccentric orbit. However, also in this scenario this source would present some peculiar properties, compared to the (admittedly few) other systems of this kind. The large variations seen in September 2003 are difficult to explain if the source was far from periastron, where no big changes in the shock properties are expected. Rotation powered pulsars in interacting binaries, such as the already mentioned PSR B1259-63 (Chernyakova et al. 2006, 2009) or the "black widow" pulsar PSR B1957+20 (Huang \& Becker 2007), have $\mathrm{X}$-ray efficiencies in the range $10^{-4}-10^{-2}$. On the other hand, the $\dot{P}$ reported by (Kaaret et al. 2000), corresponding to a rotational energy loss $\dot{E}_{\text {rot }}$ larger than a few $10^{38} \mathrm{erg} \mathrm{s}^{-1}$, implies a much lower efficiency for SAX J0635+0533.

\section{Conclusions}

Despite SAX J0635+0533 was found by XMM-Newton in a very low luminosity state, thanks to the good sensitivity of the EPIC instrument, we could derive a precise localization that confirms the proposed Be optical counterpart of this source. We observed large flux variability when the source was detected in September/October 2003 and derived a stringent upper limit of $3 \times 10^{32} \mathrm{~d}_{5}^{2} \mathrm{erg} \mathrm{s}^{-1}$ for its luminosity when the source was not detected.

The spectral and flux properties of SAX J0635+0533 are consistent with a variable neutron-star binary powered by accretion from the Be companion or by the loss of rotational energy. Both interpretations imply some peculiarities with respect to other known sources. These result from the very short spin period and possibly high period derivative reported for SAX J0635+0533, which unfortunately we could not confirm owing to the source faintness during our observations.

Acknowledgements. This work is based on observations obtained with XMM-Newton, an ESA science mission with instruments and contributions directly funded by ESA Member States and NASA. The XMM-Newton data analysis is supported by the Italian Space Agency (ASI). The Guide Star Catalogue II is a joint project of the Space Telescope Science Institute and the Osservatorio Astronomico di Torino. The Digitized Sky Surveys were produced at the Space 
Telescope Science Institute under U.S. Government grant NAG W-

2166.

\section{References}

Campana, S., Colpi, M., Mereghetti, S., Stella, L., \& Tavani, M. 1998, A\&A Rev., 8, 279

Campana, S., Stella, L., Mereghetti, S., \& Colpi, M. 1995, A\&A, 297, 385

Chernyakova, M., Neronov, A., Aharonian, F., Uchiyama, Y., \& Takahashi, T. 2009, astro-ph/0905-3341

Chernyakova, M., Neronov, A., Lutovinov, A., Rodriguez, J., \& Johnston, S. 2006, MNRAS, 367, 1201

Cusumano, G., Maccarone, M. C., Nicastro, L., Sacco, B., \& Kaaret, P. 2000, ApJ, 528, L25

Huang, H. H. \& Becker, W. 2007, A\&A, 463, L5

Johnston, S., Manchester, R. N., Lyne, A. G., et al. 1992, ApJ, 387, L37

Kaaret, P., Cusumano, G., \& Sacco, B. 2000, ApJ, 542, L41

Kaaret, P., Piraino, S., Halpern, J., \& Eracleous, M. 1999, ApJ, 523, 197

La Palombara, N. \& Mereghetti, S. 2006, A\&A, 455, 283

—. 2007, A\&A, 474, 137

Lasker, B. M., Lattanzi, M. G., McLean, B. J., et al. 2008, AJ, 136,735

Nicastro, L., Gaensler, B. M., \& McLaughlin, M. A. 2000, A\&A, 362, L5

Reig, P. \& Roche, P. 1999, MNRAS, 306, 100

Skinner, G. K., Bedford, D. K., Elsner, R. F., et al. 1982, Nature, 297, 568

Strüder, L., Briel, U., Dennerl, K., et al. 2001, A\&A, 365, L18

Thompson, D. J., Bertsch, D. L., Dingus, B. L., et al. 1995, ApJS, 101, 259

Turner, M. J. L., Abbey, A., Arnaud, M., et al. 2001, A\&A, 365, L27

Watson, M. G., Schröder, A. C., Fyfe, D., et al. 2009, A\&A, 493, 339 